\title{
On Lagrange Multipliers in Work with Quality and Reliability Assurance
}

\section{Vidal, Rene Victor Valqui; Becker, P.}

Published in:

I E E E Journal on Selected Areas in Communications

Publication date:

1986

Document Version

Publisher's PDF, also known as Version of record

Link back to DTU Orbit

Citation (APA):

Vidal, R. V. V., \& Becker, P. (1986). On Lagrange Multipliers in Work with Quality and Reliability Assurance. I E E E Journal on Selected Areas in Communications, 4(7), 1143-1148. http://www2.imm.dtu.dk/pubdb/p.php?3692

\section{General rights}

Copyright and moral rights for the publications made accessible in the public portal are retained by the authors and/or other copyright owners and it is a condition of accessing publications that users recognise and abide by the legal requirements associated with these rights.

- Users may download and print one copy of any publication from the public portal for the purpose of private study or research.

- You may not further distribute the material or use it for any profit-making activity or commercial gain

- You may freely distribute the URL identifying the publication in the public portal

If you believe that this document breaches copyright please contact us providing details, and we will remove access to the work immediately and investigate your claim. 


\title{
On Lagrange Multipliers in Work with Quality and Reliability Assurance
}

\author{
RENÉ VICTOR VALQUI VIDAL AND PETER W. BECKER, SENIOR MEMBER, IEEE
}

\begin{abstract}
In optimizing some property of a system, reliability say, a designer usually has to accept certain constraints regarding cost, completion time, volume, weight, etc. The solution of optimization problems with boundary constraints can be helped substantially by the use of Lagrange multipliers techniques (LMT). With representative examples of increasing complexity, the wide applicability of LMT is illustrated. Two particular features are put in focus. First, an easy to follow yet powerful new graphical approach is presented. Second, the concept of Fuller-Polya maps is shown to be helpful in the areas of sales promotion and teaching. These maps illuminate the logic structure of solution sequences. One such map is shown, illustrating the application of LMT in one of the examples.
\end{abstract}

\section{INTRODUCTION}

L AGRANGE multipliers techniques, LMT, have been used successfully to solve constrained optimization problems in both engineering and economics. In the field of reliability engineering, use of LMT is well documented by Tillman, Hwang, and Kou [1]. LMT's are fundamental to the applied mathematics field best known as optimization theory or mathematical programming (see Vidal [2]). However, few engineers appear to be familiar with the wide applicability of LMT; hence this paper.

During the past twenty years some effort has been made to obtain a better interaction between the theory and practice of optimization. This paper is offered as a modest contribution in this direction.

We present the LMT by introducing some fundamental concepts of optimization theory. Some elementary problems are solved emphasizing the geometrical understanding of the solution procedure. The limitations of the classical LMT are discussed.

This paper presents a new approach, a graphical (nonalgorithmic) method, to solve a family of simple resource-allocation problems. The method is illustrated within the context of a simple reliability problem.

The LMT can be displayed in the method problem structure mapping, developed by Fuller from a suggestion by Polya [3]. The Fuller-Polya map is a mechanism for systemizing and organizing the problem solving procedure. It shows the structure of the solution procedure independent of the mathematical manipulations. It focuses the attention on the solution method rather than on the specific answer. Once constructed the Fuller-Polya map

Manuscript received December 23, 1985; revised May 28, 1986

The authors are with the Technical University of Denmark, DK-2800 Lyngby, Denmark.

IEEE Log Nümber 8609901

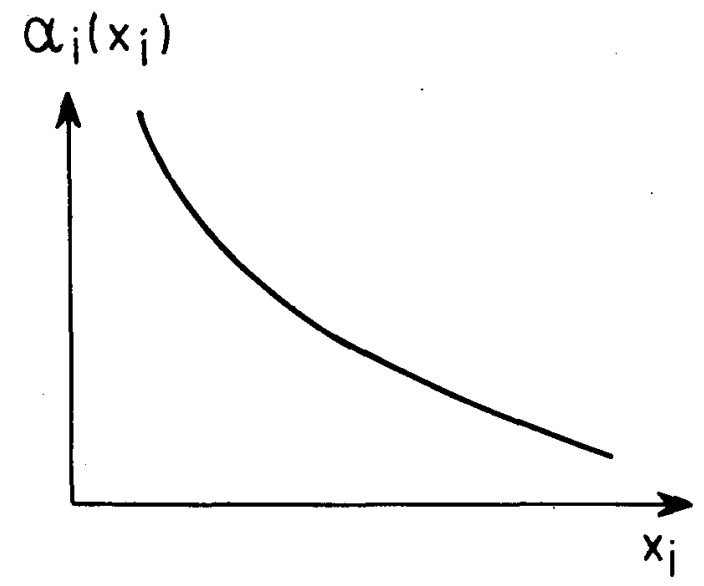

Fig. 1. Failure rate, $\alpha_{i}\left(x_{i}\right)$, versus coolant flow, $x_{i}$, for the $i$ th subsystem.

also shows the structure of the solution of all similar problems. The last section of this paper shows a Fuller-Polya diagram for an optimization problem solved by LMT.

Some important aspects of LMT are not discussed in this paper. One of these is the case of the geometrical interpretation of the optimal conditions of a constrained optimization problem resulting in the well-known KuhnTucker conditions (see Vidal [2]).

\section{Optimal Distribution of a Coolant to a Cooling System}

The LMT will be applied in the analysis of a cooling system to determine the optimum distribution of coolant to the subsystems for maximum system reliability [4]. The subsystems are assumed to be cooled in series. Only the cooling of electronic equipment will be considered. Fig. 1 shows a typical curve of failure rate, $\alpha_{i}\left(x_{i}\right)$, of the $i$ th subsystem, $i=1, \cdots, n$, as a function of the coolant flow rate, $x_{i}$.

The reliability of the $i$ th subsystem is assumed to decrease exponentially with time: $R_{i}(t)=\exp \left[-\alpha_{i}\left(x_{i}\right) t\right]$. Assuming that all subsystems are statistically independent and connected in series (no redundancy is allowed) the system reliability is

$$
R(t)=\prod_{i=1}^{n} R_{i}(t)=\exp \left[-t\left(\sum_{i=1}^{n} \alpha_{i}\left(x_{i}\right)\right)\right] .
$$

Maximizing $R(t)$ is equivalent to maximizing $\ln [R(t)]=$ $F(W)$ : 


$$
F(W)=\max \left(-\sum_{i=1}^{n} \alpha_{i}\left(x_{i}\right)\right)
$$

subject to

$$
\sum_{i=1}^{n} x_{i}=W
$$

$n$ is the number of subsystems and $W$ is the total flow rate allowed.

This constrained optimization problem can be transformed into an unconstrained one by introducing a $\mathrm{La}$ grange multiplier, $\lambda$; that is we introduce a penalty for not satisfying (2).

The unconstrained optimization problem now takes the form:

$$
\max _{x_{i}} L(x, \lambda)=-\sum_{i=1}^{n} \alpha_{i}\left(x_{i}\right)+\lambda\left(W-\sum_{i=1}^{n} x_{i}\right)
$$

The problem can be decomposed into $n$ similar problems each in one variable, $x_{i}$,

$$
\max L_{i}\left(x_{i}\right)=\left[-\alpha_{i}\left(x_{i}\right)-\lambda x_{i}\right] .
$$

If $\alpha_{i}\left(x_{i}\right)$ is a differentiable convex function, as for example, $k_{i} / x_{i}$, then a necessary and sufficient condition for optimality is

$$
-\frac{d \alpha_{i}\left(x_{i}\right)}{d x_{i}}=\lambda .
$$

Equation (3) illustrates a well-known principle in microeconomics, which states that the marginal utility of each subsystem is equal to a particular constant, the Lagrange multiplier. For the case $\alpha_{i}\left(x_{i}\right)=k_{i} / x_{i}$, (3) specializes to (4),

$$
x_{i}=\sqrt{\frac{k_{i}}{\lambda}}=f_{i}(\lambda)
$$

The $n$ values can be substituted into (2) which is satisfied for $\lambda=\lambda^{*}$.

$$
\lambda *=\frac{\left(\sum_{i=1}^{n} \sqrt{k_{i}}\right)^{2}}{W^{2}} .
$$

By specializing (4) for $\lambda=\lambda^{*}$, we obtain the optimal solution

$$
x_{i}^{*}=\frac{\sqrt{k_{i}}}{\sum_{i=1}^{n} \sqrt{k_{i}}} W, \text { i.e., } \quad \alpha_{i}\left(x_{i}^{*}\right)=\frac{\sqrt{k_{i}}}{W} \cdot \sum_{i=1}^{n} \sqrt{k_{i}}
$$

The perturbation function, (1), becomes

$$
F(W)=-\frac{\left(\sum_{i=1}^{n} \sqrt{k_{i}}\right)^{2}}{W}
$$

This function expresses the negative of the optimal total failure rates as a function of the total flow rate, $W ; F(W)$ is a concave differentiable function. Recalling (5), it is verified that

$$
\frac{d F(W)}{d W}=\lambda
$$

A popular interpretation of $\lambda$ is that of a shadow price; a shadow price is defined as the marginal increase (or decrease) in utility due to a marginal increase (or decrease) in resource.

In this example, the optimal solution could be found analytically using the LMT. In [5] a similar approach is utilized to optimize the reliability of some simple circuit problems.

The $n$ constraints $x_{i} \geq 0$ are not explicitly included in (1) and (2), but due to the structure of the problem they will automatically be satisfied.

\section{The Geometric Argument}

Let our starting point be the $n$ functions $\alpha_{i}^{\prime}=-\alpha_{i}\left(x_{i}\right)$ all of which are differentiable, strictly concave and increasing functions; see fourth quadrant of Fig. 2. Minus one times the slope of the failure rate function is called $s_{i}$ $=-d \alpha_{i}\left(x_{i}\right) / d x_{i}$; see first quadrant of Fig. 2. Next, we draw the straight line $y_{i}=\lambda x_{i}, \lambda>0$; see first quadrant of Fig. 2.

The (negative) distance, $L_{i}\left(x_{i}\right)$, from $\alpha_{i}^{\prime}$ to $\lambda_{i}$ is

$$
L_{i}\left(x_{i}\right)=-\alpha_{i}\left(x_{i}\right)-\lambda x_{i}
$$

For a given value of $\lambda, L\left(x_{i}\right)$ has its maximum (i.e., numerically speaking the distance is as short as possible) at the point $x_{i}^{*}(\lambda)$ as shown in Fig. 2, at this point the slope of $\alpha_{i}^{\prime}, s_{i}$, happens to be $\lambda$. For the $(n-1)$ other $\alpha_{i}^{\prime}$ functions we may likewise find a $x_{i}^{*}(\lambda)$-value. Obviously, these $x_{i}^{*}(\lambda)$-values yield the solution to our problem, (1) and (2), for $W=W^{*}(\lambda)$, where

$$
W^{*}(\lambda)=\sum_{i=1}^{n} x_{i}^{*}(\lambda)
$$

and the perturbation function becomes

$$
F\left(W^{*}(\lambda)\right)=\sum_{i=1}^{n}\left[-\alpha_{i}\left(x_{i}^{*}(\lambda)\right)\right] .
$$

That is, the arbitrarily chosen $\lambda$-value thus turns out to be the Lagrange multiplier value which maximizes the sum of $\alpha_{i}$-values for $W=W^{*}(\lambda)$. By choosing smaller and smaller $\lambda$-values we can consequently find larger and larger maximas, the $F\left(W^{*}(\lambda)\right)$ 's, for larger and larger $W^{*}(\lambda)$-values.

As illustrated in Fig. 2, first quadrant, the $x_{i}^{*}(\lambda)$-value may also be found as the intersection between the horizontal line $s_{i}=\lambda$ and the curve $s_{i}=-d \alpha_{i}\left(x_{i}\right) / d x_{i}$, this is the condition stipulated in (3). If we add the $n s_{i}$-curves horizontally, the sumcurve, $S$, will also be intersected by the horizontal line, this time at $W^{*}(\lambda)$. The sumcurve, $S$, 


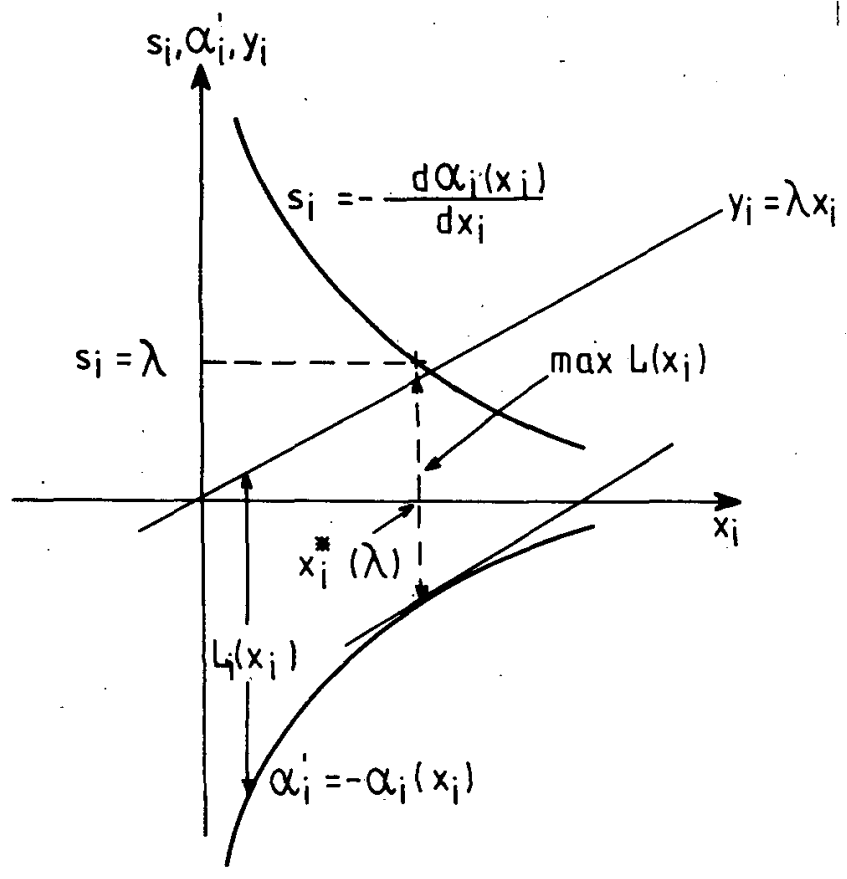

Fig. 2. The graph of $s_{i}, \alpha_{i}^{\prime}$, and $y_{i}$.

TABLE I

SEVEn $\alpha_{i}\left(x_{i}\right)$-Functions, AND TheIr Transform:

$$
x_{i}=A_{i}+B_{i} \cdot \theta(\lambda)
$$

$A_{i}$ AND $B_{i}$ ARe Functions of the $\alpha_{i}\left(x_{i}\right)$-Parameter Values, and INDEPENDENT OF THE $\lambda$-VALUE; $\theta(\lambda)$ IS A FUNCTION OF THE ACTUAL $\lambda$-VAlue, and INDEPendent of the Value of $i$.

\begin{tabular}{|llll|}
\hline$a_{i}\left(x_{i}\right)$ & $\theta(\lambda)$ & $A_{i}$ & $B_{i}$ \\
\hline $\begin{array}{l}p_{i}\left(1-k_{i} / x_{i}\right) \\
p_{i}, k_{i}>0\end{array}$ & $\frac{1}{\sqrt{\lambda}}$ & 0 & $\sqrt{p_{i} k_{i}}$ \\
\hline $\begin{array}{l}-p_{i} x_{i}^{k} \\
p_{i}>0, k>2\end{array}$ & $\lambda^{i /(k-1)}$ & 0 & $\frac{1}{\left(-p_{i} k\right)^{1 /(k-1)}}$ \\
\hline $\begin{array}{l}p_{i} /\left(a_{i}-x_{i}\right) \\
p_{i}, a_{i}>0\end{array}$ & $\frac{1}{\sqrt{\lambda}}$ & $a_{i}$ & $-\sqrt{p_{i}}$ \\
\hline $\begin{array}{l}p_{i} l o g_{e}\left(1+m_{i} x_{i}\right) \\
p_{i}, m_{i}>0\end{array}$ & $\frac{1}{\lambda}$ & $-\frac{1}{m_{i}}$ & $p_{i}$ \\
\hline $\begin{array}{l}p_{i} x_{i} /\left(a_{i}+x_{i}\right) \\
p_{i}, a_{i}>0\end{array}$ & $\frac{1}{\sqrt{\lambda}}$ & $-p_{i}$ & $\sqrt{p_{i} a_{i}}$ \\
\hline $\begin{array}{l}2 p_{i} \sqrt{x_{i}} \\
p_{i}>0\end{array}$ & $\frac{1}{\lambda^{2}}$ & 0 & $p_{i}^{2}$ \\
\hline $\begin{array}{l}p_{i}\left(1-e^{\left.-k_{i} x_{i}\right)}\right. \\
p_{i}, k_{i}>0\end{array}$ & $\ln (\lambda)$ & $\frac{\ln \left(p_{i} k_{i}\right)}{k_{i}}$ & $-\frac{1}{k_{i}}$ \\
\hline
\end{tabular}

once it haš been generated, can be used with any number of $\lambda$-values.

Generating a sumcurve, clearly, is particularly simple if all the added curves are straight lines or piecewise linear functions. As pointed out by Vidal [6] such linear relationships may be achieved by a suitable transformation whenever (3) may be solved for $x_{i}$ and given the form: $x_{i}$ $=\boldsymbol{A}_{i}+\boldsymbol{B}_{i} \cdot \theta(\lambda)$.

Seven cases are listed in Table I [6]. More often than not $\lambda^{*}$ can only be determined by numerical methods, and not analytically; e.g., with inventory problems [7] and allocation of cooling resources [8].

\section{Economic Fuel Dispatch}

In this section, we examine a more difficult problem. It is desired to minimize the total fuel consumption for a given configuration of a power generation system composed of $n$ units. The total power production of the system should be equal to a load $L$. Moreover, the $i$ th unit's output $x_{i}(\mathrm{MW})$ is subjected to a set of special constraints that impose an upper bound and a lower bound on each $x_{i}$. Assuming that the fuel consumption at each unit is a quadratic function of $x_{i}$, the optimization model is

$$
F(L)=\max _{x_{i}}\left[-\sum_{i=1}^{n}\left(a_{i}+b_{i} x_{i}+c_{i} x_{i}^{2}\right)\right]
$$

subject to:

$$
\begin{aligned}
\sum_{i=1}^{n} x_{i} & =L \\
L B_{i} & \leq x_{i} \leq U B_{i}, \quad i=1,2, \cdots, n .
\end{aligned}
$$

This problem is also decomposable, and for each $i$ we have to

$$
\max _{x_{i}}\left[-\left(a_{i}+b_{i} x_{i}+c_{i} x_{i}^{2}\right)-\lambda x_{i}\right]
$$

subject to:

$$
L B_{i} \leq x_{i} \leq U B_{i} .
$$

If the optimal $x_{i}, x_{i}^{*}$, is an interior point then

$$
d\left\{\max _{x_{i}}\right\} / d x_{i}=0
$$

for

$$
x_{i}^{*}=-\frac{b_{i}+\lambda}{2 c_{i}}
$$

if $x_{i}^{*}<L B_{i}$, reset $x_{i}^{*}=L B_{i}$, and if $x_{i}^{*}>U B_{i}$ reset $x_{i}^{*}=$ $U B_{i}$.

Now, an iterative procedure can be developed. For a given $\lambda,(13)$ uniquely determines a set of $x_{i}^{*}$ 's, if (11) is satisfied, then we have an optimal solution. Otherwise, $\lambda$ should be suitably modified. This is actually the essence of the LMT, which also can be applied to more complex problems; e.g., in [9] a fuel dispatch problem is handled by this approach. Note that the approach suggested in Section III can be modified to solve the problem in this section, since (13) already defines a linear relationship like those from Table I.

Does the LMT always work? Let us look at a third example.

\section{Least Cost Allocations of Reliability INVESTMENTS}

The problem is one of optimizing the redundancy of an $n$-stage system; each stage consists of a number $x_{i}$ of par- 
allel (redundant) components of cost $c_{i}$ and reliability $a_{i}$. The separate stages are taken to be in series, so that the system is operable if, and only if, every stage contains at least one operable component. The allocation problem is then to choose the stage redundancies $\left(x_{i}{ }^{\prime} s\right)$ in such a manner as to maximize the system reliability subject to constrained total cost. The states of all components are assumed to be statistically independent. The system's reliability is given by

$$
\prod_{i=1}^{n}\left[1-\left(1-a_{i}\right)^{x_{i}}\right]
$$

Since maximizing the logarithm of a function maximizes the function, we take our payoff to be the logarithm of the reliability. Our problem is to determine

$$
F(C)=\max _{x_{i}}\left[\sum_{i=1}^{n} \ln \left[1-\left(1-a_{i}\right)^{x_{i}}\right]\right]
$$

subject to:

$$
\begin{gathered}
\sum_{i=1}^{n} c_{i} x_{i}=c \\
x_{i} \geq 1 \quad \text { (integer), } \quad i=1,2, \cdots, n .
\end{gathered}
$$

This optimization problem differs from the two discussed earlier in that the $x_{i}$ 's ought to be integers. The (generalized) LMT can be applied in a similar way as shown above. The problem is decomposable, so we have to

$$
\max \left[\ln \left[1-\left(1-a_{i}\right)^{x_{i}}\right]-\lambda c_{i} x_{i}\right]
$$

subject to:

$$
x_{i} \geq 1 \text { (integer). }
$$

For given $\lambda$, this problem is readily solved, for instance by simple enumeration.

Now, the iterative procedure developed in the last section can also be applied to solve (14), (15), and (16). That is, $\lambda$ is modified iteratively until the values of the $x_{i}$ 's satisfy (15).

Does such a $\lambda$-value that generate the $x_{i}$ 's always exist? The answer is no. The examples we have shown in the earlier sections are so-called "nice" problems because: 1) the variables to be found were continuous, 2) the payoff function to be maximized was concave, and 3) the region of feasible values was convex. In such situations the perturbation function, $F()$, is concave and the existence of $\lambda^{*}$ corresponds to the existence of support hyperplanes; this is illustrated in Fig. 3. For the example in Section V the perturbation function is illustrated in Fig. 4.

Fig. 4 illustrates that for the problem of (14) a support hyperplane cannot always be found. This corresponds to the fact that, in general, there exists no $\lambda$ which can generate the right-hand side of (15). These regions of $C$-values are denominated gaps. Fortunately, for large-scale problems the gaps are small, and near-optimal solutions can be found, as shown in (10). The LMT as applied to

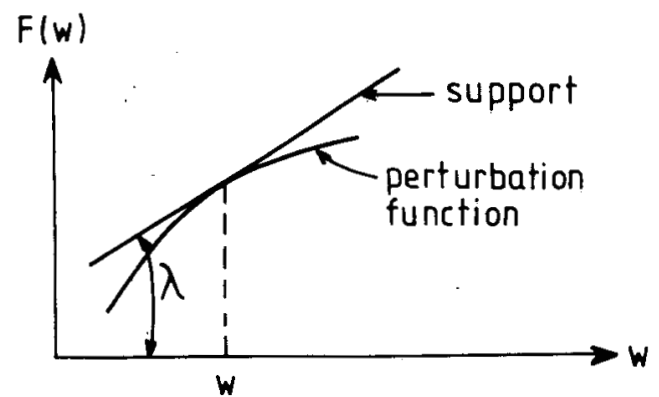

Fig. 3. Perturbation function for a "nice" problem, see (7).

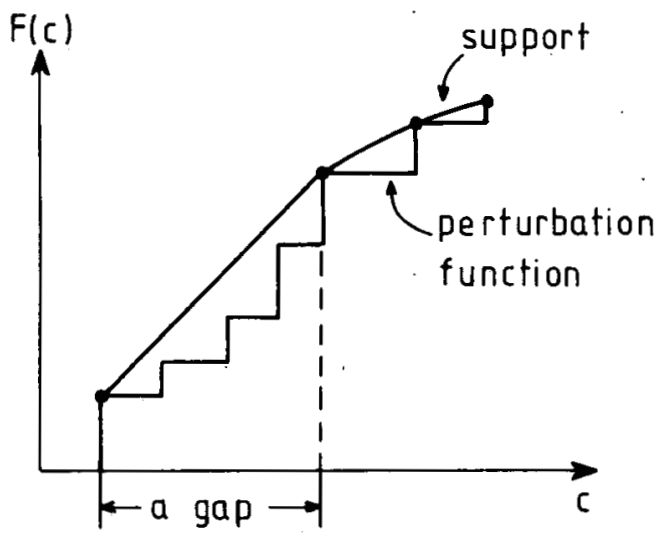

Fig. 4. Perturbation function for the problem in Section V.

the field of optimization are usually referred to as Lagrange relaxation.

\section{Nonlinear Lagrangian Functions}

Many optimization problems in engineering are not "nice," and often the problem to be solved might have many gaps. The LMT can be further generalized to work with support functions instead of support hyperplanes. This is equivalent to generalizing the classical Lagrangian function (linear in $\lambda$ )

$$
L(x, \lambda)=f(x)-\sum_{i=1}^{m} \lambda_{i} g_{i}(x)
$$

for the problem $\left\{\max _{x} f(x) \mid g_{i}(x) \leq 0, i=1,2, \cdots\right.$, $m\}$, to a generalized Lagrangian function

$$
L(x, \gamma)=f(x)-\sum_{i=1}^{m} \gamma_{i}\left(g_{i}(x)\right)
$$

to be maximized. The idea is still the same to transform a constrained problem into a sequence of unconstrained problems. Many forms of the functions $\gamma_{i}()$ have been suggested, see for instance [2]. The authors have good experiences with a quadratic function

$$
\gamma_{i}\left(g_{i}(x)\right)=a_{i}\left(g_{i}(x)\right)^{2}+\lambda_{i} g_{i}(x)
$$

to solve engineering problems.

The iterative procedure will be similar to the LMT, but we have now two multipliers $\left(a_{i}, \lambda_{i}\right)$ for each constraint. The main disadvantage resides in the fact that the generalized Lagrangian function is not decomposable. 


\section{Optimal Control Problems}

LMT can also be used to solve dynamic optimization problems [14]. Let us see a simple example, an optimal control problem. A student beginning a course which lasts $n$ weeks wants to plan his study time to pass the course without working more than necessary. His knowledge level $k$ increases as the work invested, $x$, increases; it is assumed that $\Delta k=a x^{\beta}, a>0,0<\beta<1$. However, his memory is imperfect; from one week to the next he retains only a fraction $b(0<b<1)$ of his knowledge. If $k_{i}$ is his knowledge level at the end of the $i$ th week and $x_{i}$ is the work done during the $i$ th week, then

$$
k_{i}=a \cdot x_{i}^{\beta}+b \cdot k_{i-1}, \quad i=1,2, \cdots, n
$$

The student wishes to minimize the total work invested

$$
F(K)=\max _{x_{i}}\left[-\sum_{i=1}^{n} x_{i}\right]
$$

subject to:

$$
k_{i}-a x_{i}^{\beta}-b k_{i-1}=0, \quad i=1,2, \cdots, n
$$

and

$$
k_{o}=0, \quad k_{n}=K
$$

$k_{o}$ is the initial knowledge level and $K$ is the level requirement to pass the examination. This problem is a generalization of the one discussed in [11] for $\beta=\frac{1}{2}$.

We use $n$ multipliers, $\lambda_{i}$, one per constraint. The (linear) Lagrangian function $L$ is

$$
L(x, k, \lambda)=\sum_{i=1}^{n}\left[-x_{i}-\lambda_{i}\left(k_{i}-a x_{i}^{\beta}-b k_{i-1}\right)\right] ;
$$

Note that the problem is decomposable. $L$ has to be maximized with respect to $x_{i}$ and to $k_{i}$.

$$
\begin{gathered}
\partial L / \partial x_{i}=0 \text { for: } x_{i}=\left(a \beta \lambda_{i}\right)^{1 /(1-\beta)}, \\
i=1,2, \cdots, n . \\
\partial L / \partial k_{i}=0 \text { for: } \lambda_{i}=b \lambda_{i+1}=0, \\
i=1,2, \cdots, n .
\end{gathered}
$$

Consequently,

$$
x_{i+1}^{*}=x_{i}^{*} \cdot b^{1 /(\beta-1)}, \quad i=1,2, \cdots, n-1 .
$$

That is, the student's weekly work should be increased by the constant factor $Q=b^{1 /(\beta-1)}$ each week. The optimal work schedule is completely determined from the boundary conditions $k_{o}=0$ and $k_{n}=K$.

$$
\begin{aligned}
F(K) & =-x_{1}-Q \cdot x_{1}-\cdots-Q^{n-1} \cdot x_{1} \\
& =-x_{1} \cdot\left[Q^{n}-1\right] /[Q-1] .
\end{aligned}
$$

A formal application of the LMT to optimal control problems will result in the well-known (linear) maximum principle as shown in [12]. Moreover, in [13] a generalized (nonlinear) maximum principle is shortly discussed.

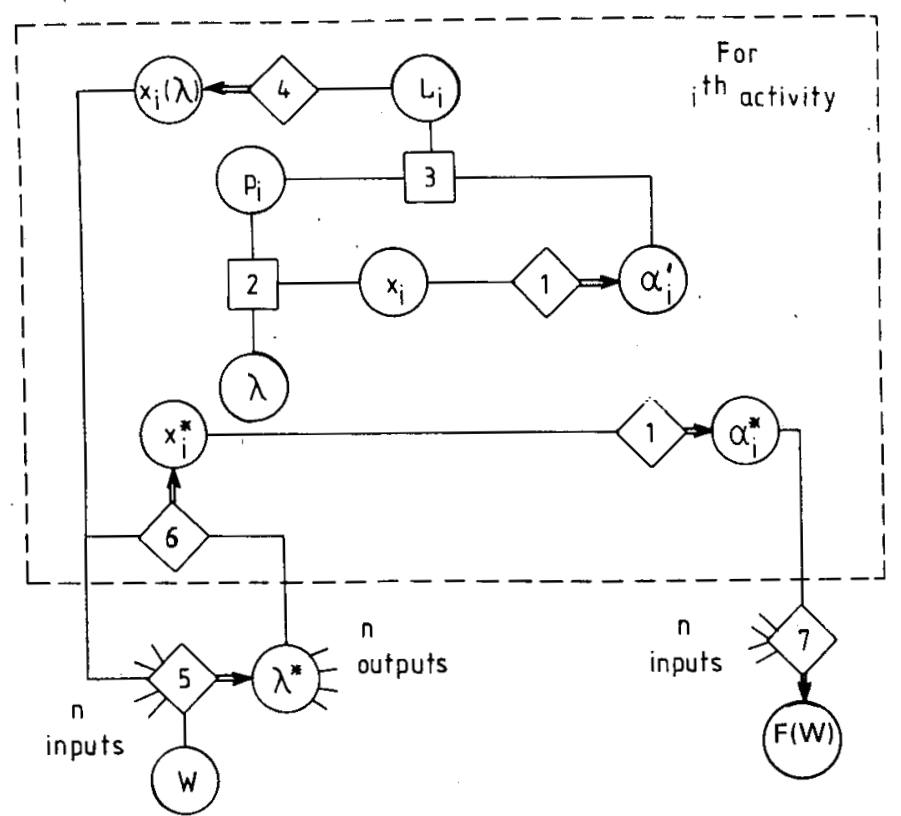

Fig. 5. Fuller-Polya map for the decomposable problem from Section II. The variables are drawn as circles (入); the algorithms are identified by number; completely reversible algorithms are shown as 3 ; irreversible (or nearly so) algorithms are shown as $\hat{0}$.

$$
\begin{aligned}
\text { 1) } \alpha_{i}^{\prime} & =\alpha_{i}\left(x_{i}\right) \\
\text { 2) } p_{i} & =\lambda x_{i} \\
\text { 3) } L_{i} & =\alpha_{i}^{\prime}-p_{i} \\
\text { 4) } \frac{d L_{i}}{d x_{i}} & =0 \Rightarrow x_{i}(\lambda)
\end{aligned}
$$

5) $\sum_{i=1}^{n} x_{i}(\lambda)=W \Rightarrow \lambda^{*}$

6) $x_{i}^{*}=x_{i}\left(\lambda^{*}\right)$

7) $F(W)=\sum_{i=1}^{n}-\alpha_{i}^{\prime *}$

These new results may be applied to a number of practical engineering problems.

\section{Constructing a Fuller-Polya Map}

Following Kardos [3], the preliminaries to construction of a Fuller-Polya map are the same as for any problem. The problem must first be understood. The data must be identified, the unknown expressed explicitly; and the physical and economical principles, with their mathematical algorithms must be identified. This is done to the best of one's abilities; but it does not need to be completed right away. The construction of the Fuller-Polya map itself will demonstrate if there are sufficient connections among the known data and the unknown quantities, and also indicate what may be missing.

Let us assume that all closed form problem solution structures have two sets of components: 1) variables (data, intermediate, solution); 2) algorithms, which related the variables to one another (they may be reversible) and/or table look-ups (which usually are irreversible).

The Fuller-Polya map for the complete problem is con- 
structed by joining the algorithms together by the way of their variables, each variable appearing only once.

Fig. 5 shows the Fuller-Polya map for the decomposable problem solved in Section II by the LMT. The elaboration of these maps within the context of optimization can be quite powerful tools in teaching and sales-promoting activities.

\section{ACKNOWLEDGMENT}

The authors would like to thank Dr. M. Kip for helpful advice.

\section{REFERENCES}

[1] F. A. Tillman, C. Hwang, and N. Kuo, Optimization of Systems Reliability. New York: Dekker, 1980, pp. 179-183.

[2] R. V. V. Vidal, "Notes on static and dynamic optimization," Inst. Mathematical Statistics and Operations Res., The Technical Univ. of Denmark, 1984.

[3] G. Kardos, "Problem solving with the Fuller-Polya diagram," to be published in Int. J. Appl. Eng. Education.

[4] R. H. Myers, L. K. Wong, and H. M. Gordy, Reliability Engineering for Electronic Systems. New York: Wiley, 1964, pp. 31-33.

[5] B. J. Brock, "A circuit analogy for reliability block diagrams," 1980 Proc. Annual Reliability and Maintainability Symp., pp. 356-362.

[6] R. V. V. Vidal, "A graphical method to solve a family of allocations problems," European J. Oper. Res., vol. 17, pp. 31-34, 1984.

[7] W. Woodside, "A multiple-commodity inventory problem with storage constraint," Int. J. Math. Educ. Sci. Technol., vol. 15, no. 1, pp. $1-5,1984$.

[8] F. Altoz, J. P. Brach, and D. Rosen, "Reliability optimization-A method for thermal design," 1982 Proc. Annual Reliability and Maintainability Symp., pp. 303-308.

[9] F. J. Trefny and K. Y. Lee, "Economic fuel dispatch," IEEE Trans. Power Apparatus and Syst., vol. PAS-100, no. 7, pp. 3468-3477, July 1981 .

[10] H. Everett, III, "Generalized Lagrange multiplier method for solving problems of optimum allocation of resourcer," Oper. Res., vol. 11, pp. $399-417,1963$.

[11] W. Woodside, "Lagrange multipliers for engineers-Some applications," Int. J. Appl. Eng. Ed., vol. 1, pp. 61-64, 1985; or, "A student optimal control problem (or how to pass courses with the minimum expenditure of effort)," App. Math. Notes, vol. 7, pp. 1-13, 1982.

[12] R. V. V. Vidal, "On the sufficiency of the linear maximum principle," to be published in J. Optimization Theory and Applications.
[13] Z. Nahorski, H: Ravn, and R. V. V. Vidal, "The discrete-time maximum principle-A survey and some new results," Int. J. Control, vol. 40, no. 3, pp. 533-554, 1984.

[14] P. W. Becker and F. Jensen, Design of Systems and Circuits for Maximum Reliability or Maximum Production Yield. New York: McGraw-Hill, 1977, appendix II.

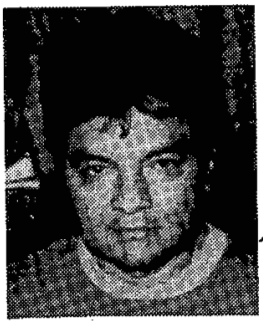

René Victor Valqui Vidal was born in Peru, where he received the M.Sc. degree in mechanical engineering from the National University of Engineering, Lima, Peru, in 1965. He took graduate courses in operations research, mathematical statistics, organization, and econometrics at the $\mathrm{Fa}-$ culté Polytechnique de Mons, Belgium, during 1966. In 1970, he presented his Ph.D. dissertation, "Operations Research in Production Planning," at the Technical University of Denmark.

He currently is an Associate Professor of Operations Research at The Technical University of Denmark. During the last 5 years, he has been working with optimization and optimal control theory and has published two books and 30 articles in international journals.

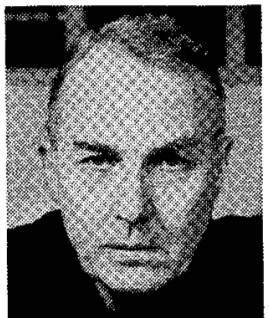

Peter W. Becker (SM'78) received the M.Sc. and Dr.Techn. degrees in electrical engineering from the Technical University of Denmark, in 1950 and 1968, respectively.

Since 1967, he has been a member of the staff at the Electronics Laboratory, Technical University of Denmark, where he teaches reliability theory and pattern recognition theory. Dr. Becker.has written several papers in the field of reliability. In 1977, he published the book Design of Systems and Circuits for Maximum Reliability or Maximum Production Yield, coauthored by F. Jensen. He is also interested in pattern recognition and has written a number of papers in that field. In 1968 he published the book Recognition of Patterns Using the Frequencies of Occurrence of Binary Words; a third, revised and enlarged edition appeared in 1978. In 1972, he published the book An Introduction to the Design of Pattern Recognition Devices.

Dr. Becker is on the Editorial Board of the International Journal of General Systems. He was First Vice-President, International Association for Pattern Recognition from 1980 to 1982. 